\title{
INTERACTION-DRIVEN ASYMMETRIC COEXISTENCE CURVES AND THE SINGULAR DIAMETER
}

\author{
James S. WALKER \\ Department of Physics, Washington State University, Pullman, WA $99164-2814$, USA \\ and \\ Raymond E. GOLDSTEIN \\ Laboratory of Atomic and Solid State Physics, Cornell University, Ithaca, NY 14853, USA
}

Received 22 May 1985; accepted for publication 6 August 1985

\begin{abstract}
The interplay of asymmetric interactions and density fluctuations in binary liquid mixtures is shown to produce a breakdown of the law of rectilinear diameter. We find that this singularity and the overall phase diagram asymmetry are suppressed with increasing pressure. These considerations suggest the systems in which the diameter anomaly should be most readily observed.
\end{abstract}

The coexistence curves of virtually all phase-separating binary liquid mixtures are asymmetric under interchange of the two components, reflecting their underlying microscopic dissimilarity. Basic chemical considerations show that this lack of component-exchange symmetry arises from differences both in molecular sizes and in the energies of interactions. Despite these asymmetries, theory and experiment [1] show that critical solution points in binary mixtures are in the universality class of the Ising ferromagnet, which has "up-down" symmetry at criticality. While this model is a suitable starting point in studying such mixtures, it is clear that to understand the complexities of real systems one must introduce degrees of freedom beyond those which merely describe substitution of one component for the other.

At the most fundamental level, a complete macroscopic description of a binary mixture requires three thermodynamic fields. These are conventionally taken to be temperature, pressure, and the chemical potential difference between the two components $A$ and $B$. In the usual mixture magnet correspondence, however, in which all lattice sites are occupied by particles of one species or the other, density fluctuations are left out, as if the pressure were infinite. In that limit, and within the approximation to our model described below, energetic asymmetries serve only to redefine the component chemical potential, and do not result in phase diagram asymmetry.

In this letter, then, we study a microscopic lattice hamiltonian which reveals in detail a mechanism by which interaction-energy asymmetry leads to coexistence-curve asymmetry when the constraint of infinite pressure is relaxed. As first suggested by Widom and Rowlinson [2], the diameter of a coexistence curve (i.e., the locus of tie-line midpoints) may, in addition to deviating from the line of compositional symmetry, exhibit a singular temperature dependence. Subsequent model calculations [3] and renormalization-group studies [4] have confirmed this prediction, and a thermodynamic argument suggests its generality [5]. Yet, this singularity has defied conclusive experimental verification [6], and has not been explained theoretically from a microscopic viewpoint. The model studied here ties the amplitude of the singularity to a microscopic description of the interactions in the system and to density fluctuations, and in so doing suggests the systems in which the anomaly should be most readily observed.

The density fluctuations in a binary liquid mixture 
can be modeled by introducing vacancies into the usual Ising model description, thus the full Hamiltonian we study is

$$
-\beta \mathscr{K}=\sum_{\langle i j\rangle} V_{i j} t_{i} t_{j}+\sum_{i}\left[h s_{i}+\Delta\right] t_{i}
$$

where $\beta=1 / k_{\mathrm{B}} T$, and the first sum is over nearest neighbors (nn) on a cubic lattice. The "vacancy" variables $t_{i}=0$ (vacant), 1 (occupied) are controlled by the chemical potential $\Delta$, while the "compositional" variables $s_{i}= \pm 1(\mathrm{~A}, \mathrm{~B})$ are governed by the field $h$. Pairwise interactions between occupied sites are denoted by $V_{i j}$.

Of most relevance to this work are the many aqueous mixtures of polar organic molecules which exhibit closed solubility loops, primarily due to entropic effects associated with intermolecular hydrogen bonding. We describe this complex miscibility using the ideas embodied in the Walker-Vause (WV) hamiltonians $[7,8]$, which describe both general and specific features of solubility phenomena in a variety of systems $[9,10]$.

Molecular orientational degrees of freedom in the WV models are given a discrete representation by the use of $q$-state Potts spins [11], $\sigma_{i}=1, \ldots, q$, so that the pair interaction $V_{i j}$ can be written as $V_{i j}=V_{i j}^{\mathrm{A}}$ $+V_{i j}^{S}$, a sum of symmetric and asymmetric terms with

$$
\begin{aligned}
& V_{i j}^{\mathrm{S}}=K_{1}\left(1-\delta_{s_{i} s_{j}}\right) \delta_{\sigma_{i} \sigma_{j}}+K_{2}\left(1-\delta_{s_{i} s_{j}}\right)\left(1-\delta_{\sigma_{i} \sigma_{j}}\right) \\
& \quad+K_{3} \delta_{s_{i} s_{j}} \delta_{\sigma_{i} \sigma_{j}}, \\
& V_{i j}^{\mathrm{A}}=\frac{1}{2} h_{1}\left(s_{i}+s_{j}\right) \delta_{\sigma_{i} \sigma_{j}}+\frac{1}{2} h_{2}\left(s_{i}+s_{j}\right)\left(1-\delta_{\sigma_{i} \sigma_{j}}\right) .
\end{aligned}
$$

In the above $\delta$ is the Kronecker delta. In the symmetric limit, $V_{i j}^{\mathrm{A}}=0$, the pair interaction reduces to that studied previously [9]; in particular, $K_{1}$ represents the hydrogen bonding interaction between unlike molecules, $K_{3}$ is that for the like-molecule bonding and $K_{2}$ is the unlike-molecule van der Waals energy. The only symmetry-breaking fields in the hamiltonian, aside from the chemical potential $h$, are the field-like splittings $h_{1}$ and $h_{2}$, which act on the like-molecule bonding and nonbonding levels, respectively.

We study the present model by a partial-trace approximation derived previously [8], similar to those of a mean field theory of orientational interactions [12]. The former consists of an exact summation over the non-critical degrees of freedom (orientational and density fluctuations) on a single-bond graph, mapping the original model onto the Ising model with an effective nn coupling and magnetic field. The reduced free energy per site $f=-\beta F / N$ in this approximation is

$f\left(\left\{J_{i}\right\}\right)=g\left(\left\{J_{i}\right\}\right)+f_{\mathrm{I}}\left(K_{\mathrm{I}}\left(\left\{J_{i}\right\}\right), H_{\mathrm{I}}\left(\left\{J_{i}\right\}\right)\right)$,

where $g$ is a smooth function of the original couplings and fields $\left(\left\{I_{i}\right\}=K_{1}, K_{2}, K_{3}, h, h_{1}, h_{2}, \Delta\right)$, and $K_{\mathrm{I}}$, $H_{\mathrm{I}}$ are the effective coupling and magnetic field which enter into the free energy $f_{\mathrm{I}}$ of the Ising model. The coexistence condition, $H_{1}=0$, determines the bare chemical potential, $h$, in terms of the remaining microscopic variables. The mole fractions of A molecules in the left-hand $(-)$ and right-hand $(+)$ branches of the coexistence curve are $X_{ \pm}=(1 \pm m) / 2$, with the spontaneous magnetization of the Ising spins $m=\left\langle s_{i}\right\rangle=\partial f /$ $\partial h$ obtained from eq. (3):

$X_{ \pm}=\frac{1}{2}\left[1+\left(\partial g / \partial h+e_{\mathrm{I}} \partial K_{\mathrm{I}} / \partial h \pm m_{\mathrm{I}} \partial H_{\mathbf{I}} / \partial h\right)_{\text {coex }}\right]$

Here, $e_{\mathrm{I}}=\partial f_{\mathrm{I}} / \partial K_{\mathrm{I}}$ is the nearest neighbor correlation $d\left\langle s_{i} s_{j}\right\rangle$, and $m_{\mathrm{I}}=\partial f_{\mathrm{I}} / \partial H_{\mathrm{I}}=\left\langle s_{i}\right\rangle$ the magnetization of the Ising model in the limit $I_{\mathrm{I}} \rightarrow 0^{+}$.

The degree of asymmetry in a coexistence curve is reflected in the deviation from one half of the diameter, which is given by $X_{d}=\left(X_{+}+X_{-}\right) / 2$. In our approximation,

$X_{\mathrm{d}}=\frac{1}{2}\left[1+\left(\partial g / \partial h+e_{\mathrm{I}} \partial K_{\mathrm{I}} / \partial h\right)_{\text {coex }}\right]$.

Clearly, in order for asymmetry to appear, the temperature-like quantities $g$ and $K_{\mathrm{I}}$ must depend on the bare one-body field $h$. This mixing of thermodynamic fields is the essence of the decorated-lattice calculations which predict a singular diameter [3], and also appears in the "revised" scaling equation of state proposed by Mermin and Rehr [5]. The breakdown of the law of rectilinear diameter arises from that part of the internal energy of the Ising model, $e_{\mathrm{I}}$, which varies as $t^{1-\alpha}$, where $\alpha$ is the specific heat exponent.

From the partial-trace approximation, we find

$K_{\mathrm{I}}=\frac{1}{4} \ln \left(Z_{1} Z_{2} / Z_{3}^{2}\right), \quad H_{\mathrm{I}}=\frac{1}{4} \ln \left(Z_{1} / Z_{2}\right)$, $g=\frac{1}{4} \ln \left(Z_{1} Z_{2}\right)-d K_{\mathrm{I}}+\Delta$,

where 

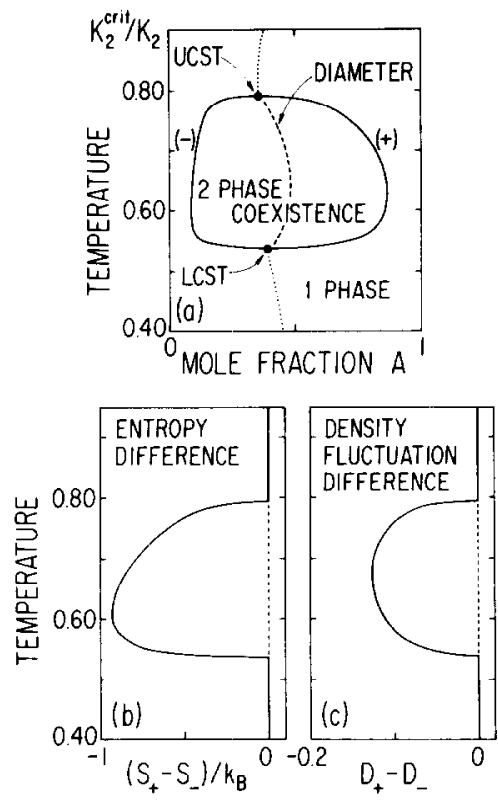

Fig. 1. A calculated asymmetric phase diagram, and relative thermodynamic properties for an A + B liquid mixture. Temperature is $T=-1 / K_{2}$, normalized to the critical coupling of the Ising model. (a) The coexistence curve (solid line) has A-rich $(+)$ and B-rich $(-)$ branches, terminating at upper (UCST) and lower (LCST) critical solution temperatures, and a diameter (dashed) which is extended into the onephase region (dotted). The difference in entropy $S$ per bond in the two branches is negative (b) arising in part from greater density fluctuations in the $(-)$ branch (c).

$Z_{1}=s^{2}\left[q u^{2} z+q(q-1) v^{2}\right]+2 q^{2} r^{-1} s+q^{2} r^{-2}$, $Z_{2}=s^{-2}\left[q u^{-2} z+q(q-1) v^{-2}\right]+2 q^{2} r^{-1} s^{-1}+q^{2} r^{-2}$, $Z_{3}=q x+q(q-1) y+q^{2} r^{-1}\left(s+s^{-1}\right)+q^{2} r^{-2}$, and $x=\mathrm{e}^{K_{1}}, y=\mathrm{e}^{K_{2}}, z=\mathrm{e}^{K_{3}}, r=\mathrm{e}^{\Delta}, s=\mathrm{e}^{h}, u=\mathrm{e}^{h_{1}}$, $v=\mathrm{e}^{h_{2}}$.

Fig. 1 illustrates the results of a calculation for a system with a closed-loop coexistence curve, and in which the interactions between AA pairs are energetically favored over the corresponding $\mathrm{BB}$ interactions (i.e., $h_{1}, h_{2}>0$ ). A qualitative understanding of the observed asymmetry can be obtained by considering interactional correlations in the system, that is, spin correlations conjugate to the various couplings and fields $\left\{J_{i}\right\}$. These derivatives of the free energy have the same form as eq. (4) for the order parameter conjugate to $h$. In particular, the entropy difference (per bond) between the coexisting phases is
$\left(S_{+}-S_{-}\right) / k_{\mathrm{B}}=(-2 / d) m_{\mathrm{I}} \sum_{i} J_{i} \partial H_{\mathrm{I}} / \partial J_{i}$

The essential result we find for a variety of systems is that the phase rich in the more strongly interacting component, say $\mathbf{A}$, has a lower energy of mixing, while the other, less correlated phase, has more mixing and orientational entropy (fig. 1b). In addition, the extent of local density fluctuations, as measured by the quantities $D_{ \pm}=1-\left\langle t_{i}\right\rangle_{ \pm}$(fig. $1 \mathrm{c}$ ), is grcater in the B-rich phase. Thus, as a consequence of the asymmetries of the interactions, one phase has a larger effective coordination number than the other, even though all sites of the lattice are equivalent. This result should be contrasted with decorated-lattice calculations ${ }^{\neq 1}$ [3] of asymmetric coexistence curves and the singular diameter, in which the static structure of the lattice gives rise to broken compositional symmetry because of the presence of two inequivalent sets of sites with different coordination numbers.

In the limit of no density fluctuations $(\Delta \rightarrow+\infty)$, both $g$ and $K_{\mathrm{I}}$ become independent of $h$, and the coexistence curve is completely symmetric - independent of any asymmetries in the microscopic interactions. Of course, the diameter is temperature-independent in this case. This infinite-pressure symmetry is also seen in the van der Waals equation of state of a binary mixture, and the spin-1 model for two-component systems [13]. The absence of asymmetry in this limit may be an artifact of our approximation, but may be a more general characteristic of lattice-gas models of particles with orientational interactions, and the issue merits further study. Even so, we still find that the entropic and correlation asymmetries discussed above remain in this limit, a consequence of the temperature dependence of the coexistence surface in thermodynamic-field space.

The singularity in the diameter of an asymmetric coexistence curve can clearly be seen in fig. $1 \mathrm{a}$ as the cusp where the diameter meets its mathematical continuation into the one-phase region. Our investigations show that the magnitude of the diameter anomaly increases with the vacancy concentration. It thus appears that a binary mixture whose critical solution temperature is very near the liquid-vapor transition is likely

${ }^{* 1}$ The thermodynamic properties of the three-dimensional Ising model were computed using the Pade approximants ref. [13]. 
to display a large anomaly, and we conjecture that it is the small compressibility of most binary liquid mixtures which has limited the observability of the singularity. The results also suggest that the phase diagrams typical of hydrogen-bonded systems, in which there are large energetic asymmetries associated with hydrogen bonding and van der Waals interactions, may be better candidates for observation of the singularity than many of the systems studied to date. In addition, the strong pressure dependence of the miscibility curve in such systems suggests the important role of vacancies. Calculations of the observability of the singularity in various types of binary mixtures will be reported elsewhere, along with a full exposition of the model.

We have benefited from stimulating discussions with N.W. Ashcroft, A. N. Berker, E.D. Glandt, N.D. Mermin, J.J. Rehr, C.A. Vause, and B. Widom. R.E.G. acknowledges the support of a graduate fellowship from the Fannie and John Hertz Foundation, and the hospitality of the Department of Physics at Washington State University. J.S.W. thanks Washington State University for its continuing support. This work was also supported in part by N.S.F. under Grant No. DMR83-14625 at Cornell University.

\section{References}

[1] A. Kumar, H.R. Krishnamurthy and E.S.R. Gopal, Phys. Rep. 98 (1983) 57, and references thercin.

[2] B. Widom and J.S. Rowlinson, J. Chem. Phys. 52 (1970) 1670.

[3] N.D. Mermin, Phys. Rev. Lett. 26 (1971) 957.

[4] J.F. Nicoll, Phys. Rev. A24 (1981) 223.

[5] N.D. Mermin and J.J. Rehr, Phys. Rev. Lett. 26 (1971) 1155 .

[6] S.C. Greer, B.K. Das, A. Kumar and E.S.R. Gopal, J. Chem. Phys. 79 (1983) 4545.

[7] J.S. Walker and C.A. Vause, Phys. Lett. A79 (1980) 421; J. Chem. Phys. 79 (1983) 2660.

[8] C.A. Vause and J.S. Walker, Phys. Lett. 90A (1982) 419.

[9] R.E. Goldstein and J.S. Walker, J. Chem. Phys. 78 (1983) 1492.

[10] R.E. Goldstein, J. Chem. Phys. 79 (1983) 4439.

[11] R.B. Potts, Proc. Cambridge Philos. Soc. 48 (1952) 106.

[12] R.E. Goldstein, J. Chem. Phys. 83 (1985) 1246.

[13] P.E. Scesney, Phys. Rev. B1 (1970) 2274.

[14] G.R. Andersen and J.C. Wheeler, J. Chem. Phys. 69 (1978) 3403.

[15] P.H. van Konynenburg and R.L. Scott, Phil. Trans. R. Soc. 298 (1980) 495; J. Sivardiere and J. Lajzerowicz, Phys. Rev. A11 (1975) 2090. 